Using the ISS to Prepare for Exploration (01)

ISS as the Foundation for Exploration (1)

Author: Mrs. Kim Ess

National Aeronautics and Space Administration (NASA), United States, kim.m.ess@nasa.gov

Dr. Harley Thronson

National Aeronautics and Space Administration (NASA)/Goddard Space Flight Center, United States, harley.a.thronson@nasa.gov

Mr. Mark Boyles

Jet Propulsion Laboratory - California Institute of Technology, United States, mark.a.boyles@jpl.nasa.gov

Dr. William Sparks

Space Telescope Science Institute, United States, sparks@stsci.edu

Dr. Marc Postman

Space Telescope Science Institute, United States, postman@stsci.edu

\title{
USING THE ISS AS A TESTBED TO PREPARE FOR THE NEXT GENERATION OF SPACE-BASED TELESCOPES
}

\begin{abstract}
The ISS provides a unique opportunity to develop the technologies and operational capabilities necessary to assemble future large space telescopes that may be used to investigate planetary systems around neighboring stars. Assembling telescopes in space is a paradigm-shifting approach to space astronomy. Using the ISS as a testbed will reduce the technical risks of implementing this major scientific facility, such as laser metrology and wavefront sensing and control (WFSC). The Optical Testbed and Integration on ISS eXperiment (OpTIIX) will demonstrate the robotic assembly of major components, including the primary and secondary mirrors, to mechanical tolerances using existing ISS infrastructure, and the alignment of the optical elements to a diffraction-limited optical system in space. Assembling the optical system and removing and replacing components via existing ISS capabilities, such as the Special Purpose Dexterous Manipulator (SPDM) or the ISS flight crew, allows for future experimentation and repair, if necessary. First light on ISS for OpTIIX, a small 1.5 meter optical telescope, is planned for 2015. In addition to demonstration of key risk-retiring technologies, the OpTIIX program includes a public outreach program to show the broad value of ISS utilization.
\end{abstract}

